\title{
Design and Development of Multi-purpose Combination Tool for Testing
}

\section{Voice Cable}

\author{
Shu Jiang ${ }^{1, a}$, Zhe Sun ${ }^{1, b}$, Qiang $\mathrm{Li}^{1, \mathrm{c}}$ and Ying Zhang ${ }^{2, \mathrm{~d}}$ \\ ${ }^{1}$ State Grid Chang Chun Power Supply Company, Chang chun 130000, China; \\ ${ }^{2}$ Jilin Province Economic Management Cadre College, Chang chun 130000, China. \\ ajiangshu2004@sohu.com, b46878299@qq.com, ${ }^{\mathrm{c}} 306755518 @ q q . c o m,{ }^{\mathrm{d}} 78264005 @ q q . c o m$.
}

Keywords: Voice distribution frame, testing tools, multi-purpose combination tool.

\begin{abstract}
This paper introduces the necessity of using the tools for testing the cable of voice distribution frame. A detailed comparison of existing voice cable testing tools is provided, a design scheme of multi-purpose combination tool for testing voice cable is provided. This paper develops a multi-purpose combination tool for testing voice cable from different perspectives, and summarizes the corresponding method of using multi-purpose combination tool for testing voice cable according to different purposes.
\end{abstract}

\section{Introduction}

In the field of power communications industry, voice distribution frame as the special frame of local telephone ${ }^{[1]}$, network and telephone board as the transfer point of terminal equipment and switching equipment, often need to be tested because of work needs. In order to complete the testing work more efficiently, the professional testing tools of cable are essential. Existing voice cable testing tools are single-purpose testing tools mostly. In general, only one type of cable of voice distribution frame can be tested, but the network and telephone board can not be tested. The test needs more steps and more time, so efficiency is low. When it is necessary to test different types voice distribution frame cable and network and telephone board, the tester must carry several testing tools to complete the work at the same time, it will increase the inconvenience of testing work.

\section{Comparison of existing voice cable testing tools}

Now, there are three kinds of cable testing tools: duckbill test line, KRONE voice module test line and alligator clip test line, as shown in Fig.1.The duckbill test line is made up of duckbill contacts, telephone line and RJ11 telephone line connector; the KRONE voice module test line is made up of KRONE voice module test contacts, telephone line and RJ11 telephone line connector; the alligator clip test line is made up of alligator clips, telephone line and RJ11 telephone line connector $^{[2]}$.The first two kinds of testing contacts are all fixed, and unadjustable, only can test the cable of TCL110 voice distribution frame and KRONE voice distribution frame respectively, can't be shared, in addition, the testing success ratio of the two kinds of testing tools is not high, for the alligator clip test line because the alligator clip is relatively wide, therefore, it only can be used to test the cable of voice distribution frame which spacing of metal terminals is relatively wide, such as Huahired, Huawei and Sunsea etc. The three kinds of tools for testing voice cable do not have the function of testing network and telephone board. In conclusion, so far there is no one kind of cable 
testing tools which can test TCL110 and KRONE voice distribution frame which spacing of metal terminals is relatively narrow, and can test Huahired, Huawei and Sunsea voice distribution frame which spacing of metal terminals is relatively wide, also can test network and telephone board at the same time.

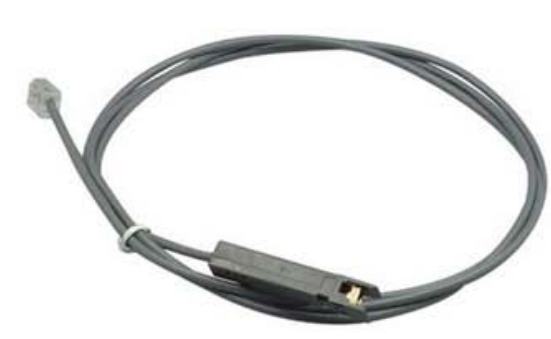

Duckbill Test Line

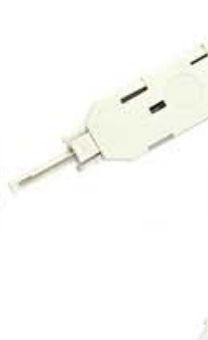

KRONE Voice Module Test Line

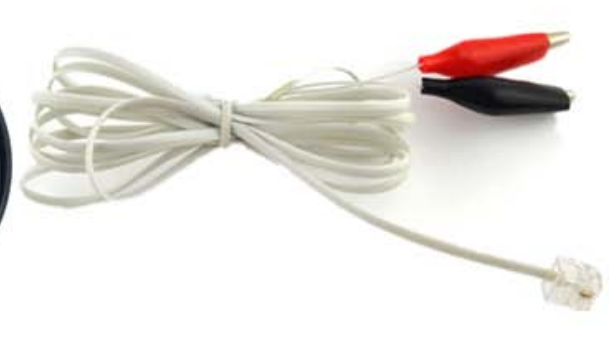

Alligator Clip Test Line

Fig.1 Existing cable testing tools

3. Design of multi-purpose combination tool for testing voice cable

By analyzing the construction and usage of voice cable testing tools in the market, in allusion to the disadvantages of single function and narrow application of these testing tools, a scheme of designing a multi-purpose combination tool for testing voice cable is proposed. Because the test contacts of duckbill test line and KRONE voice module test line are all fixed, and unadjustable, so they only can be used as a special testing tools to test the cable of a type voice distribution frame, and the function is too single. In order to resolve this problem, a design of clamp structure testing tools is proposed. It can test the cable of different types voice distribution frame by adjusting the spacing of testing tools contacts. For the clamp structure testing tools, the voice cable is tested by inserting two testing contacts in a pair of terminals of the voice distribution frame, and the spacing of different types voice distribution frame metal terminal gasket is not the same, the general range is between $0.18 \sim 2 \mathrm{~mm}$, therefore, in order to meet the requirement of testing the cable of different types voice distribution frame, it is necessary that the thickness of the contacts must be less than $0.18 \mathrm{~mm}$.According to the special requirement of testing contact, a new type of sheet contact with a thickness of only $0.15 \mathrm{~mm}$ is designed and selected. It is less than the spacing of all current types voice distribution frame metal terminal gasket, so it can meet the requirement of testing the cable of all types voice distribution frame at the same time. In order to enrich the methods of testing the cable of voice distribution frame, the paper introduces the existing alligator clip test line, and splices the alligator clip test line and the clamp structure testing tools, playing a spare role in testing. In addition, in order to test network and telephone board at the same time, a design scheme is provided, which splices the RJ11 phone head test line, the clamp structure testing tools and the alligator clip test line as a combination tool, as shown in Fig.2. 


\section{The Name Of Each Component}

\begin{tabular}{|c|l|}
\hline 1 & Testing Tools Of Clamp Structure \\
\hline 2 & Alligator Clip Test Line \\
\hline 3 & RJ11 Phone Head Test Line \\
\hline 4 & Test Phone Cable \\
\hline 5 & Test Phone \\
\hline
\end{tabular}

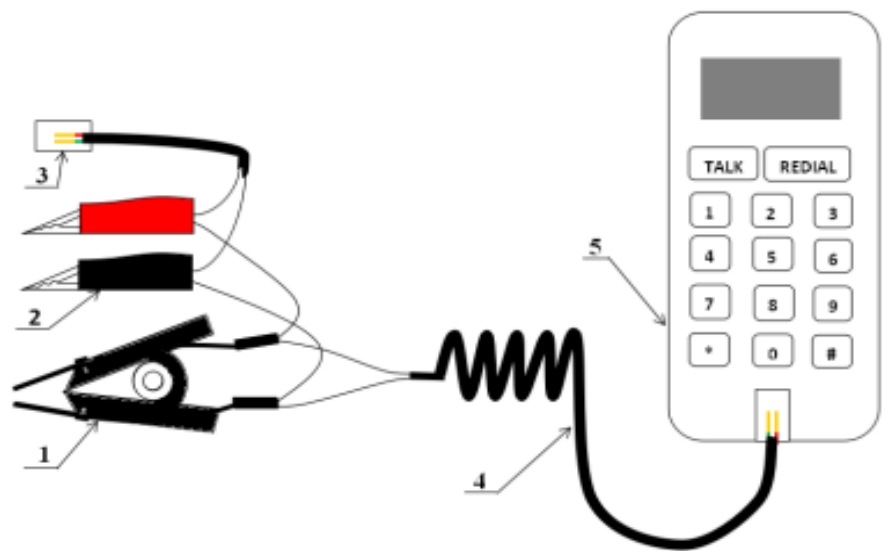

Fig.2 The design of multi-purpose combination tool

4. Development of multi-purpose combination tool for testing voice cable

According to the design scheme above proposed, considering from insulation, by comparing with the materials of the existing testing tools, the body and connectors of clamp structure testing tools are made of plastic material is more reasonable. Considering from consistency and durability, the testing contact is made of steel sheet with the length, width, thickness of $100 \mathrm{~mm}, 8 \mathrm{~mm}, 0.15 \mathrm{~mm}$. As the testing contact traverses the body is along the direction of the body of clamp structure testing tools, in order to fix the testing contact on the body of clamp structure testing tools, the screw of M1.4 is used for connection and fixation. In order to make sure the tail of testing contact and the telephone line are connected firmly, a welding way at the junction is adopted with a pyrocondensation pipe for protection and reinforcement. Considering from resisting the external pull and saving the space of cable storage, using the copper wire of two core with screwing design as the telephone line is more appropriate. The finishing multi-purpose combination tool for testing voice cable, as shown in Fig.3.

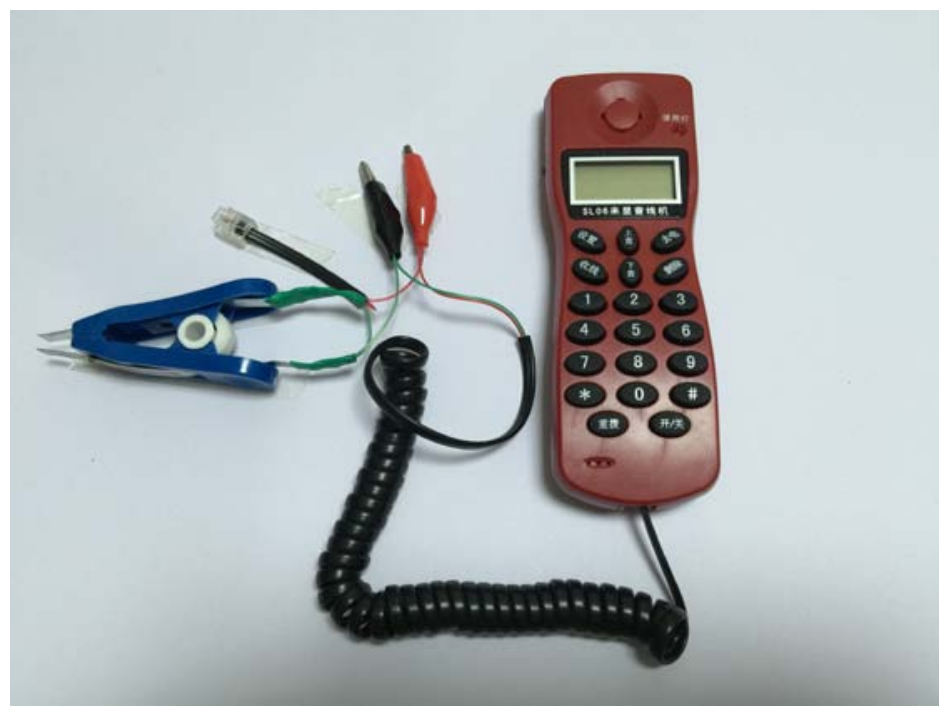

Fig.3 The real figure of multi -purpose combination tool

5. Features of multi -purpose combination tool for testing voice cable

Comparison with the existing testing tools, design and development of multi-purpose combination tool for testing voice cable in this paper, because of the clamp structure, it can be adjusted freely, and the thickness of the testing contact is only $0.15 \mathrm{~mm}$,it is less than the distance of 
all types of voice distribution frame metal terminal gasket, so it can meet the requirement of testing the cable of all types of voice distribution frame. In addition, the alligator clip test line and the RJ11 phone test line are introduced into multi-purpose combination tool, making the structure of the testing tools more reasonable, enriching the methods of testing the cable of voice distribution frame, enhancing the function of multi-purpose combination tool, broadening the purposes of multi-purpose combination tool, achieving the purpose of saving labor, time and cost.

6. The using method of multi-purpose combination tool for testing voice cable

\section{A. Continuity Test}

The continuity test of the clamp structure testing tools :turn the multimeter to the diode position [3] , contact a testing contact of the clamp structure testing tools with a probe, and contact a metal terminal of RJ11 phone head which is connected test phone with another probe. If we hear the beep, it indicates that the line is conducting. This method also can be used to test another testing contact is whether conductive.

The continuity test of alligator clip test line: the test method is the same as the continuity test of the clamp structure testing tools.

The continuity test of RJ11 phone head test line: the test method is the same as the continuity test of the clamp structure testing tools.

\section{B. Preparation for testing}

The RJ11 phone head which is connected test phone should be inserted into the test phone, the switch key of test phone should be turned to "off-hook".

\section{The steps of testing the cable of Huahired, Huawei, Sunsea and TCL110 voice distribution frame}

When the cable of Huahired, Huawei and Sunsea voice distribution frame are tested, we can use the clamp structure testing tools, also can use the alligator clip test line, but the cable of TCL110 voice distribution frame only can be tested with the clamp structure testing tools.

1) The steps of testing the cable of Huahired, Huawei, Sunsea and TCL110 voice distribution frame with alligator clip test line

Step1: First of all, the tails of the two alligator clips should be pressed respectively, so the two alligator clips can clamp a terminal with the cable respectively.

Step2: Getting the receiver of the test phone close to the ear, if the dial tone is heard, it indicates that the cable in the terminal is conductive; if the dial tone is not heard, it indicates that the cable in the terminal is interrupted.

Step3: In the state of conduction, we can use the test phone to check the telephone number of the voice cable.

2) The steps of testing the cable of Huahired, Huawei, Sunsea and TCL110 voice distribution frame with the clamp structure testing tools

Step1: First of all, the tails of the clamp structure testing tools should be pressed, when the spacing of two testing contacts of the testing tools is properly adjusted, they are inserted in a pair of terminals with the cable.

Step2: Getting the receiver of the test phone close to the ear, if the dial tone is heard, it indicates that the cable in the terminal is conductive; if the dial tone is not heard, it indicates that the cable in the terminal is interrupted.

Step3: In the state of conduction, we can use the test phone to check the telephone number of the voice cable, as shown in Fig.4. 


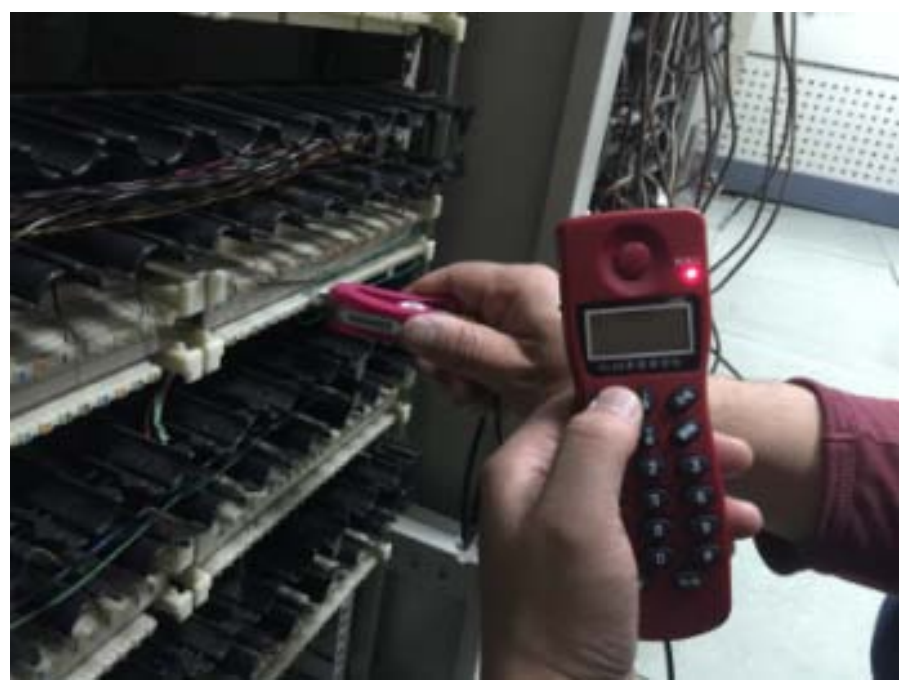

Fig.4 Testing the cable of TCL110 with the testing tool of clamp structure

$D$. The steps of testing the port of network and telephone board with the RJ11 phone head test line

Step1: The RJ11 phone head should be inserted into the port of network and telephone board.

Step2: Getting the receiver of the test phone close to the ear, if the dial tone is heard, it indicates that the cable in the terminal is conductive; if the dial tone is not heard, it indicates that the cable in the terminal is interrupted.

Step3: In the state of conduction, we can use the test phone to check the telephone number of the voice cable.

\section{Summary}

By comparing the existing voice cable testing tools, for the disadvantages of single function and narrow application of these testing tools, a design scheme of multi-purpose combination tool for testing voice cable is provided. According to the design scheme, from different perspectives, the paper develops a multi-purpose combination tool for testing voice cable. Comparison with the existing voice cable testing tools, multi-purpose combination tool for testing voice cable can meet the requirement of testing the cable of all types voice distribution frame at the same time. In addition, the alligator clip test line and the RJ11 phone test line are introduced into multi-purpose combination tool, adding the function of testing network and telephone board, making the structure of the testing tools more reasonable, achieving the purpose of saving labor, time and cost. At last, summarizes the corresponding method of using multi-purpose combination tool for testing voice cable according to different purposes.

\section{References}

[1]Dajin Wu. Data compilation of equipment and materials is commonly used in communication lines[M]. The Posts and Telecommunications Press, Peking, 1995, pp.357-413.

[2]Wufei Xie, Yifan Lu, Wei Liang. Research on the convenient alligator clip of terminal board[J]. Electronics World, 2014, 20, p.476-476.

[3]Bing Zheng, $\mathrm{Xu}$ Yang. Research on the commonly detection equipment and test methods of analog circuit[J]. Science \& Technology Information, 2012, 22, p.88-88. 